\title{
The Physical Activity Leader and Comprehensive School Physical Activity Program Effectiveness
}

\author{
Timothy A. Brusseau, Ryan D. Burns \\ Department of Health, Kinesiology and Recreation, University of Utah, Salt Lake City, USA
}

\section{Summary}

Study aim: Two thirds of children are not meeting recommended levels of physical activity. A solution to physical inactivity is Comprehensive School Physical Activity Programming (CSPAP). Little is known regarding the impact of a school's physical activity leader on CSPAP effectiveness. Therefore, this study explored changes in physical activity accumulated during school hours in CSPAP schools being led by the physical education teacher compared to a stand-alone physical activity leader. Material and methods: Participants were 1325 children from ten elementary schools in the Southwest U.S. participating in a CSPAP. Children wore Yamax DigiWalker pedometers prior to CSPAP implementation and again at the end of the academic year when CSPAP was fully implemented. Five schools had physical education teachers serve as CSPAP leaders and five schools had stand-alone leaders.

Results: Results suggest that stand-alone physical activity leader schools yielded significant increases in school-day step counts from baseline to follow-up compared to physical education leader schools (mean change difference $=1901$ steps, $p<0.001$, $\mathrm{d}=0.73)$.

Conclusions: These findings may provide insight to the challenges of being a full time physical education teacher while trying to train and engage other teachers/staff in physical activity promotion. Stand-alone physical activity leaders or a committee approach to CSPAP implementation may be more effective for increasing physical activity in schools.

Keywords: Coordinated school health programs - Child/adolescent health - Health promotion Physical activity/exercise

\section{Introduction}

The health benefits of physical activity for schoolaged children are well established [26]. Unfortunately, most children do not accumulate the recommended levels of physical activity [19]. In fact, a recent study [28] exploring physical activity trends in 32 countries found that a majority of youth do not accumulate the recommended 60 minutes per day, with less than one in three meeting the guideline. In the U.S., schools have been identified as an ideal location to promote physical activity in children [23] and to combat physical inactivity. The Centers for Disease Control and Prevention [16] have recommended that schools adopt Comprehensive School Physical Activity Programs (CSPAP).

A CSPAP is a multi-component school initiative that includes (a) quality physical education, (b) physical activity during the school day (including recess and classroom opportunities), (c) activity before and after school, (d) staff involvement, and (e) parental/community engagement. Most CSPAP efforts have excluded staff involvement and parental/community involvement [24, 31]. CSPAP interventions have demonstrated positive change across a number of outcomes [4] including physical activity, health-related fitness and motor skill development. More specifically, recent research has suggested that CSPAP can improve a number of outcomes including physical activity [15], health-related fitness [3], motor skill proficiency [9], classroom on-task behavior [8] and physical activity enjoyment [20]. Kulinna and colleagues [30] reported that multi-component school physical activity programming decreased the number of school nurse visits and improved school attendance and Burns and colleagues [7] linked CSPAP to improvements in cardio-metabolic health.

Carson, Castelli, Beighle, and Erwin [12] have identified leadership as an essential level of influence for school-based physical activity programming. Specifically, 
Beighle et al. [2] identified the physical education teacher as the person who should be this leader in schools. It is important that these leaders have organization and administration skills, public health knowledge, advocacy tools and physical activity backgrounds. Carson [11] suggests that physical activity leaders need training and also highlights that this person should ideally be the physical education teacher. Heidorn and Centeio [22] identify the role of the physical activity leader to train school personnel to develop and integrate physical activity into academic curricula, provide encouragement, and create opportunities for school personnel to participate in activities themselves. Jones and colleagues [27] found that classroom teachers and school staff in addition to the physical education teacher provide leadership in CSPAP efforts suggesting that a group effort is needed to be successful. Additionally, Goh, Hannon, Webster and Brusseau [21] found that the physical education teacher has limited time to provide the necessary leadership alone to successfully implement CSPAP. With this in mind, it is important to examine the leadership structure of school CSPAP efforts with program effectiveness. It has also been noted previously that teachers need on-going professional development and this has been linked to CSPAP effectiveness [13]. Lastly, when physical activity leaders set goals for students, CSPAPs have shown greater increases in physical activity [5].

CSPAP studies have used both the physical education teacher [7] and stand-alone school staff [4] as physical activity leaders and have both had success increasing physical activity. Goh et al., [21] however, has suggested that physical education teachers find it challenging to fulfill recommended leadership responsibilities. Little effort has been made to specifically examine the role of the physical activity leaders in CSPAP success. Therefore, the purpose of this study was to examine the effectiveness of identical CSPAPs, led by the physical education teacher or a standalone physical activity leader on school-day physical activity accumulation. It was hypothesized that schools with the stand-alone physical activity leader would have greater increases in physical activity compared to CSPAPs led by physical education teachers.

\section{Material and methods}

\section{Participants}

Participants were a convenience sample of children $(\mathrm{N}=1325$; age $=9.5 \pm 1.3$ years; $655=$ girls $)$ recruited from ten elementary schools. Children were recruited from the $1^{\text {st }}$ through $6^{\text {th }}$ grades. Schools were located in a metropolitan area in the Southwest U.S. Physical education teachers and stand-alone physical activity leaders were hourly paraprofessionals, although some had physical education or physical activity training. Parental permission and student assent were collected prior to the study. All aspects of the study were approved by the University Institutional Research Board, School District Research Boards, and school principals.

\section{CSPAP}

Each of the schools implemented a similar CSPAP. Specifically, the schools ensured the physical education teachers received training on quality physical education practices as well as an activity centric curricula. Recess supervisors at all schools received training on maximizing physical activity opportunities and classroom teachers received information on both activity breaks and active academics (e.g. Take 10!; Kibbe et al., [29]). Before- and after-school physical activity opportunities included morning drop in opportunities and school sponsored after school programs. Limited staff involvement and parent/ community engagement were included.

The main difference between the schools was the physical activity leader. Five schools used their existing physical education teacher in this role. Beyond their teaching duties they provided encouragement, support, and training for other school personnel implementing physical activity. Physical education was taught one time per week for 45 minutes and recess was offered at lunchtime and either in the morning or afternoon. Classroom teachers implemented activities one to three times per day but had minimal support from the physical education teacher during class time. The other five schools had stand-alone physical activity leaders whose sole responsibility was to implement the CSPAP. The stand-alone position was a paraprofessional who had a sole responsibility of promoting physical activity across the school day. The stand-alone physical activity leader schools also had a separate paraprofessional who served as the physical education teacher. These paraprofessionals were able to assist in physical education and recess as well as work directly with classroom teachers to infuse physical activity into their classrooms. More specifically, physical education classes were offered once per week for 45 minutes. Children had a lunch recess as well as either a morning or afternoon recess. Classroom teachers implemented activity breaks between one and three times per day.

\section{Instruments}

Physical activity was assessed using the Yamax DigiWalker CW600 pedometer (Tokyo, Japan) [1]. Each student in the study wore the pedometer for one week at the beginning of the school year and again at the end of the school year when the CSPAP was fully implemented. Children wore the instrument for five days at school (Monday through Friday) from 8:00 AM to 3:00 PM. Pedometers were worn on the hip at the level of the iliac crest aligned with the right kneecap. Both shake tests and step tests 
were done ahead of both data collection time points by the research team to ensure accuracy of the instrument and the appropriate hip placement [32]. The Yamax DigiWalker is considered a valid and reliable assessment of physical activity in elementary school children [1].

\section{Procedures}

Baseline data was collected over the first 5 weeks of the school year (two schools per week. Physical activity leaders received training during the baseline data collection weeks on their roles and responsibilities and what a CSPAP is. These training took place during professional development days and were similar across all schools. CSPAP trainings for school personnel took place monthly throughout the school year. The physical activity leaders led the CSPAP implementation throughout the school year. At the end of the school year, physical activity data was collected again to determine the changes in step counts over the course of the year from the CSPAP implementation.

\section{Data analysis}

Data were screened for outliers using box-plots and zscores. Differences among grade levels, sexes, and physical activity leader conditions at the baseline time-point were examined using a $6 \times 2 \times 2$ Factorial Analysis of Variance (ANOVA) test. A Bonferroni post hoc test was employed with alpha level adjustment if a statistically significant grade level main effect was found. The primary analysis consisted of a Mixed-Design $6 \times 2 \times 2$ ANOVA test with repeated measures to examine pedometer step count differences across grade levels $\left(1^{\text {st }}\right.$ through $6^{\text {th }}$ grade), physical activity leader conditions (physical education teacher, stand-alone), and time (baseline, follow-up). It was found that there were no differences in step counts between the sexes, therefore sex was not added into the model as an additional factor. The primary effect of interest was the physical activity leader condition $\times$ time interaction, however the three-way grade level $\times$ physical activity leader condition $\times$ time interaction was also explored. All estimates were bootstrapped across 1,000 iterations to ensure precision of the estimates. Effect sizes were calculated using partial eta-squared $\left(\eta^{2}\right.$ partial $)$ for ANOVA effects and Cohen's delta (d) for pairwise comparisons. Effect sizes were considered small if $\mathrm{d}<0.20$, medium if $\mathrm{d} \approx 0.50$, and large if $\mathrm{d}>0.80$ [17]. All analyses had an a priori alpha level of $p \leq 0.05$ and were carried out using SPSS v25.0 statistical software package (Armonk, NY, USA).

\section{Results}

Two extreme baseline time-point pedometer step count outliers were identified and subsequently deleted $(\mathrm{z}>+3.5 ;<1 \%$ of total sample). At the baseline timepoint, there were significant differences among grade levels with children in the $5^{\text {th }}$ grade displaying higher step counts than children in $2^{\text {nd }}$ grade (mean difference $=699$ steps, $p<0.05, \mathrm{~d}=0.34$ ). There were no statistically significant differences in step counts between the sexes. Additionally at the baseline time-point, there were significant differences between physical activity leader conditions with children in the physical education teacher condition displaying greater step counts compared to children in the stand-alone leader group (mean difference $=2095$ steps, $p<0.001, \mathrm{~d}=0.93$ ). Figure 1 and Figure 2 display the aforementioned contrasts across physical activity leader conditions and grade levels, respectively. Figure 3 displays the interaction between PAL condition and time, which was determined to be statistically significant $(\mathrm{F}=146.1$,

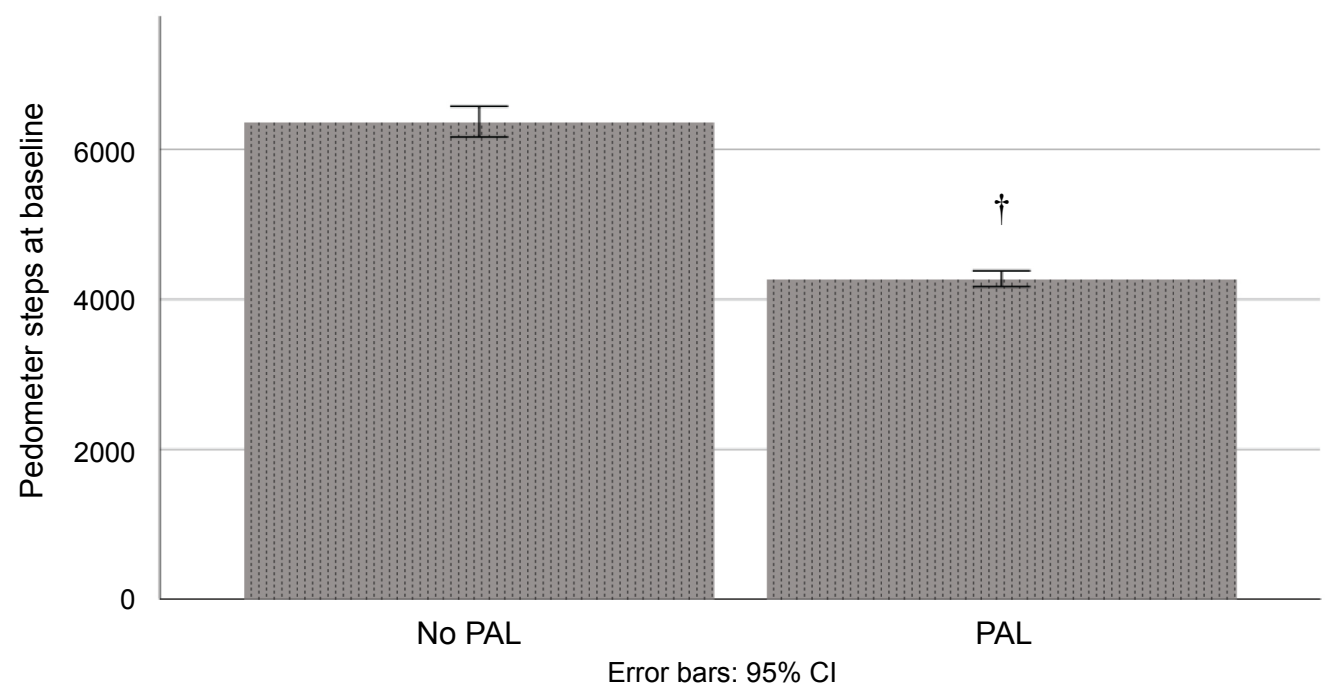

Fig. 1. Pedometer step counts across PAL conditions at baseline time-point.

PAL stands for Physical Activity Leader; error bars are 95\% Confidence Intervals; $\uparrow$ denotes statistical differences between conditions, $p<0.05$. 


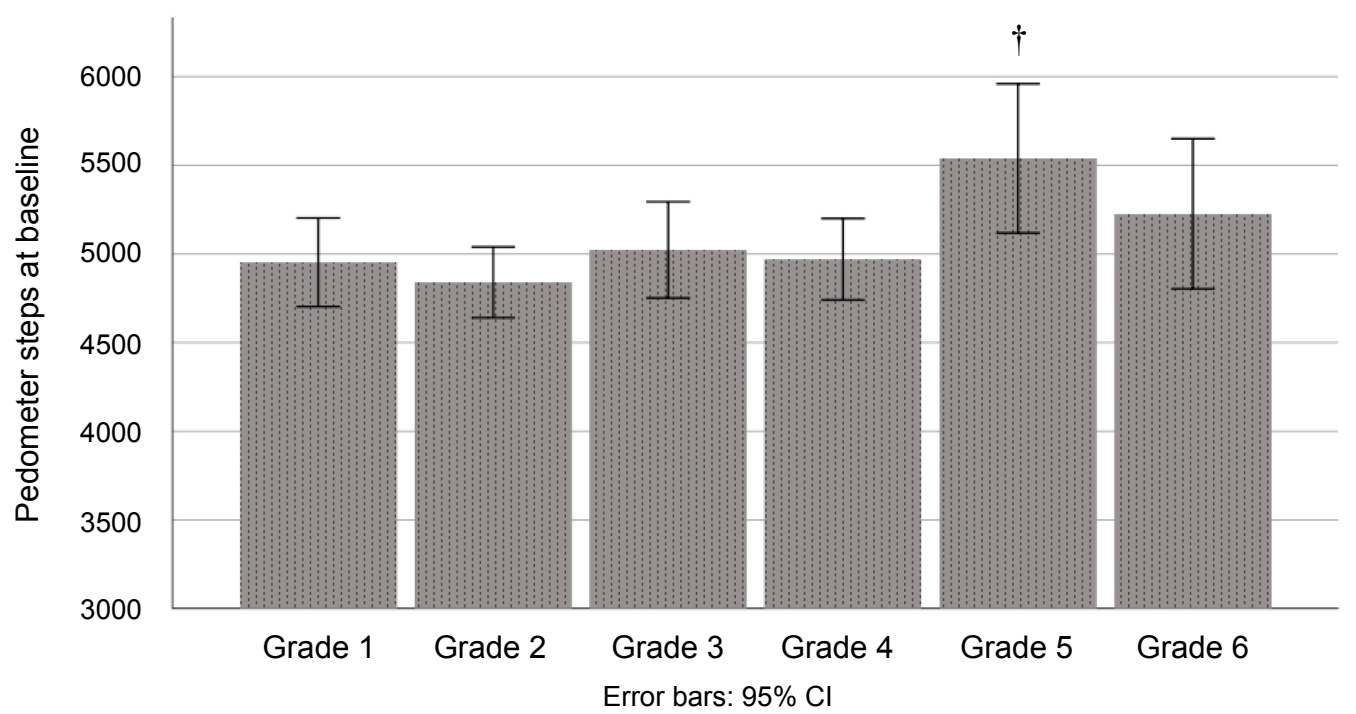

Fig. 2. Pedometer step counts across grade levels at baseline time-point.

Error bars are 95\% Confidence Intervals; $\uparrow$ denotes statistical differences compared to Grade 2, $p<0.05$.

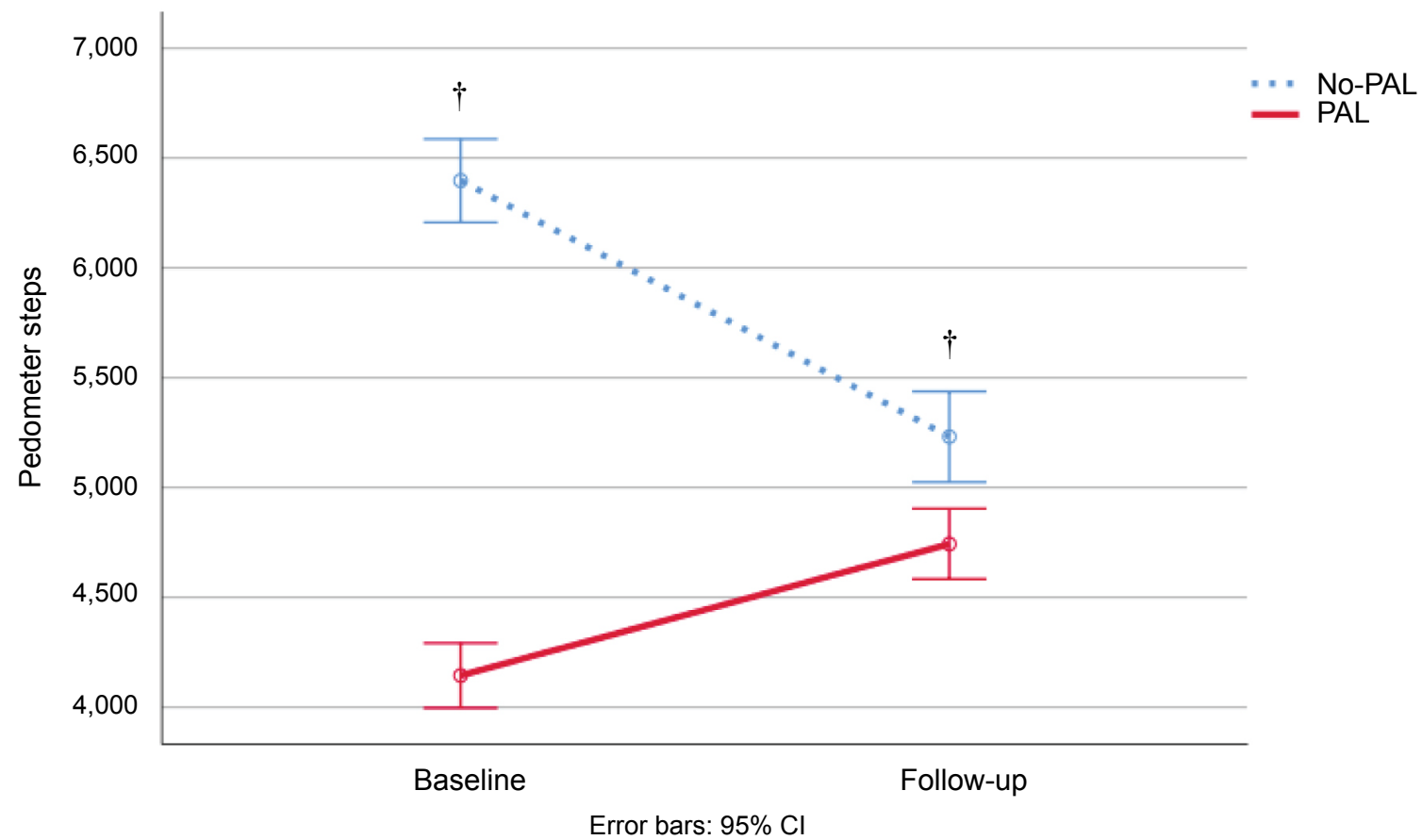

Fig. 3. Pedometer steps across time-points per PAL condition.

PAL stands for Physical Activity Leader; error bars are 95\% Confidence Intervals; $\uparrow$ denotes statistical differences between conditions, $p<0.05$.

$\left.p<0.001, \eta_{\text {partial }}^{2}=0.15\right)$. Children in the physical education leader condition decreased their steps from the baseline and follow-up time-point ( $\Delta=-1238$ steps), while students in the stand-alone leader condition increased their steps from baseline to follow-up time points $(\Delta=+663$ steps). This difference in change was statistically significant and represented a medium-to-large effect size (mean change difference $=1901$ steps, $p<0.001, \mathrm{~d}=0.73$ ) . There was no three-way grade $\mathrm{x}$ PAL condition $\mathrm{x}$ time interaction found in the analysis $(\mathrm{F}=0.69, p=0.597$, $\left.\eta_{\text {partial }}^{2}=0.003\right)$.

\section{Discussion}

The purpose of this study was to examine changes in school day step counts in CSPAP schools where the lone difference was who served as the school physical activity leader. The physical activity leader has been identified as an essential component of CSPAP planning and implementation [13]. The physical education teacher is the most qualified person for this position given their physical activity expertise [11], but given the typical teaching load of 
the physical education teacher, it may be a challenge for them to allocate the necessary time to the needed training and support of other school personnel [21]. Indeed, Goh and colleagues [21] found that physical education teacher found it difficult to fulfill their physical activity leader responsibilities when considering other teaching and supervisory expectations. This may help to explain why the physical education teacher led CSPAP did not see increases in school physical activity; whereas the stand-alone physical activity leaders had the sole responsibility to promote physical activity across the school day. They were able to assist in physical education classes and recess. More specifically, they were able to set up equipment and activity stations for semi structured recess and make changes as needed during the activity time. The physical education teacher could provide some equipment but was not present to encourage physical activity. Similarly, the stand-alone physical activity leaders could teach classroom teachers how to implement classroom activity opportunities or even lead some activities. Again, the physical education teacher could only access the classroom teachers before or after school or on in-service training days.

Interestingly, the physical education teacher led CSPAP schools were significantly more active both at baseline and intervention time points when compared to the stand-alone leader schools. This may be explained by the socioeconomic status and ethnic/racial makeup of the participating schools. The five physical education teacher led schools had an average of $40 \%$ of children receiving free or reduced lunch and $28 \%$ ethnic minority compared to $89 \%$ free and reduce lunch and $84 \%$ ethnic minority at the stand-alone schools. Carlson, Mignano and Norman [10] suggest that low socioeconomic schools are less likely to have quality physical activity supportive practices and that higher socioeconomic schools had 4.4 more minutes of moderate to vigorous physical activity when compared to lower socioeconomic status schools. Taken together, this may have explained the baseline differences and is supported by the fact that the physical education teachers at the stand-alone physical activity leader schools were not certified specialists and in fact were hourly staff. There was however, a significant decrease from baseline in the physical education led schools. This may be partly attributed to the fact that the children in these schools were already very active. Since students were already achieving recommended levels, there may not have been much room for improvement. The Institute of Medicine [24] recommends that children should accumulate 30 minutes of moderate to vigorous physical activity while attending school. Burns and colleagues [6] suggest that 30 minutes of MVPA is accumulated in 5,505 steps. This number would suggest that a vast majority of students in the physical education led CSPAP schools were exceeding this recommendation.
Perhaps leaving little room for additional physical activity opportunities. Even at the intervention time point, the mean step counts were just shy of the 5505 target $(\sim 5200$ steps). The stand-alone physical activity leader schools were well short of the recommended step count levels and although they significantly increased, the students remained below the 30-minute threshold. The exact reasons for falling short of the recommendation are unknown, however, the types of physical education lessons or recess activities may have been less active than the other schools. Future research needs to contextualize the physical activity opportunities to better understand what took place during activity opportunities.

To ensure success of CSPAPs, school may need to adopt a team approach to leadership and implementation. Jones et al. [27] found that a variety of school teachers and staff have been engaged in school physical activity promotion. In the current study, the schools primarily relied on a single person to provide the vast majority of the leadership and trainings. Physical educators are the most qualified for school physical activity promotion [11], but other teachers and staff could have specific roles to assist. An example could include the assistant principal providing direct supervision/encouragement for recess paraprofessionals and a classroom teacher or two taking a leadership role in classroom physical activity endeavors. The stand-alone physical activity leader may not be someone who can be covered by existing budgets, although pooling recess funds with before/after school funds may allow for a physical activity leader to be hired. Castelli and Beighle [14] noted that most schools should have a wellness committee and each of the members could take on a role in promoting physical activity. Additionally, the success of school change efforts are often linked to the effectiveness of the school principal [18]. Kulinna et al. [30] noted that the lack of program effectiveness in one of the schools might be linked to the lack of commitment by the school principal when compared to the other schools in their study.

The current study is not without limitations. First the study lacked a true control or comparison group, which attenuates the internal validity of the results by limiting the ability to directly link change in physical activity to the CSPAP. Second, the study was conducted in one region of one state in the US, which limits the generalizability of the study. Additional data collection time points may have been helpful to confirm increases in physical activity throughout the school year. Physical activity was assessed using pedometers; the construct validity of the results may have been stronger if accelerometers were used or energy expenditure was estimated. Finally, qualitative process information was not collected, which precludes conclusions drawn regarding quality control of CSPAP implementation across the school year. 
This was an important first look at the effectiveness of a CSPAP in relation to who served as the physical activity leader. The initial findings presented here suggest that the stand-alone physical activity leader may help with increasing school physical activity although these schools did not meet recommended levels. The physical education teacher led schools saw a decrease in physical activity although they were more active at both time points than the standalone physical activity leader schools. As Jones et al., [27] also suggests, it is likely that multiple teachers and staff are willing to be engaged in physical activity promotion. Most schools have wellness committees, and these committees, if they split up CSPAP responsibilities may be the best approach to maximize a CSPAP. Without a doubt, the physical education teacher has the most training for such a role but unfortunately, they may be spread too thin to do everything needed for a successful CSPAP. Perhaps school need to consider reallocating some of the time and effort of the physical education teacher so that they can fully implement and/or oversee the CSPAP.

\section{Conflict of interest: Authors state no conflict of interest.}

\section{References}

1. Barfield J.P., Rowe D.A., Michael T.J. (2004) Inter-instrument consistency of the Yamax Digi-Walker pedometer in elementary school-aged children. Meas. Phys. Educ. Exerc. Sci., 8: 109-116.

2. Beighle A., Erwin H., Castelli D., Ernst M. (2009) Preparing physical educators for the role of physical activity director. JOPERD, 80: 24-29.

3. Brusseau T.A., Hannon J.C., Burns R.D. (2016) Effects of Comprehensive School Physical Activity Program on physical activity and health-related fitness in at-risk youth, J. Phys. Act. Health, 13: 888-894.

4. Brusseau T.A., Hannon J.C., Fu Y., Fang Y., Nam K., Goodrum S., Burns R.D. (2018) Trends in physical activity, health-related fitness, and gross motor skills in children during a two-year comprehensive school physical activity program. J. Sci. Med. Sport, DOI:10.1016/j. jsams.2017.12.015

5. Burns R.D., Brusseau T.A., Fu Y. (2017) Influence of goal setting on physical activity and cardiorespiratory endurance in low-income children enrolled in CSPAP schools. Am. J. Health Educ., 48: 32-40.

6. Burns R.D., Brusseau T.A., Fu Y., Hannon J.C. (2016) Establishing school day pedometer step count cut-points using ROC curves in low-income children. Prev. Med., 86: 117-122.

7. Burns R.D., Brusseau T.A., Hannon J.C. (2017) Effect of Comprehensive School Physical Activity Programming on cardiometabolic health markers in children from lowincome schools. J. Phys. Act. Health, 14: 671-676.

8. Burns R.D., Brusseau T.A., Fu Y., Myrer R., Hannon J.C. (2016) Impact of CSPAP on children on-task behavior at school. Am. J. Health Behav., 40: 100-107.

9. Burns R.D., Fu Y., Fang Y., Hannon J.C., Brusseau T.A. (2017) Effect of a 12-Week physical activity program on gross motor skills in children. Percep. Mot. Skills, 124: 1121-1133.

10. Carlson J.A., Mignano A.M., Norman G.J., McKenzie T.L., Kerr J., Arredondo E.M., Sallis J.F. (2014) Socioeconomic disparities in elementary school practices and children's physical activity during school. Am. J. Health Prom., 28(3_suppl): S47-S53.

11. Carson R.L. (2012) Certification and duties of a director of physical activity. JOPERD, 83: 16-29.

12. Carson R.L., Castelli D.M., Beighle A., Erwin H. (2014) School-based physical activity promotion: A conceptual framework for research and practice. Child. Obes., 10: 100-106. DOI:10.1089/chi.2013.0134.

13. Carson R.L., Castelli D.M., Pulling KuhnA.C., Moore J.B., Beets M.W., Beighle A., Glowacki E.M. (2014) Impact of trained champions of comprehensive school physical activity programs on school physical activity offerings, youth physical and sedentary behaviors. Prev. Med., 69: S12-S19. DOI:10.1016/j.ypmed.2014.08.025.

14. Castelli D.M., Beighle A. (2007) The physical education teacher as school activity director. JOPERD, 78: 25-28.

15. Centeio E.E., Somers C., McCaughtry N., Shen B., Gutuskey L., Martin J., Kulik N.L. (2014) Physical activity change through comprehensive school physical activity programs in urban elementary schools. J. Teach. Phys. Educ., 33: 573-591. DOI: 10.1123/jtpe.2014-0067.

16. Centers for Disease Control and Prevention. (2013) Comprehensive School Physical Activity Programs: A Guide for Schools. Atlanta, GA: U.S. Department of Health and Human Services.

17. Cohen J. (1988) Statistical power analysis for the behavioral sciences.

18. Deal T.E., Peterson K.D. (2016) Shaping school culture. John Wiley \& Sons.

19. Dentro K.N., Beals K., Crouter S.E., Eisenmann J.C., McKenzie T.L., Pate R.R., Katzmarzyk P.T. (2014) Results from the United States' 2014 report card on physical activity for children and youth. J. Phys. Act. Health, 11(s1): S105-S112.

20. Fu Y., Burns R.D., Brusseau T.A., Hannon J.C. (2016) Comprehensive school physical activity programming and activity enjoyment. Am. J. Health Behav., 40: 496-502.

21. Goh T.L., Hannon J.C., Webster C., Brusseau T.A. (in press). Infusing physical activity leadership training in PETE programs through university-school partnership: principals and graduate student experiences. Phys. Educ. 
22. Heidorn B., Centeio E. (2012) The director of physical activity and staff involvement. JOPERD, 83: 13-26.

23. Hills A.P., Dengel D.R., Lubans D.R. (2015) Supporting public health priorities: Recommendations for physical education and physical activity promotion in schools. Prog. Cardio. Dis., 57: 368-374.

24. Hunt K., Metzler M. (2017) Adoption of Comprehensive School Physical Activity Programs: A literature review. Phys. Educ., 74: 315.

25. Institute of Medicine (2013) Educating the Student Body: Taking Physical Education to School. The National Academies Press.

26. Janssen I., LeBlanc A.G. (2010) Systematic review of the health benefits of physical activity and fitness in schoolaged children and youth. Int. J. Beh. Nutr. Phys. Act., 7: 40 .

27. Jones E.M., Taliaferro A.R., Elliott E.M., Bulger S.M., Kristjansson A.L., Neal W., Allar I. (2014) Chapter 3 Feasibility study of comprehensive school physical activity programs in Appalachian communities: The McDowell CHOICES project. J. Teach. Phys. Educ., 33: 467-491.

28. Kalman M., Inchley J., Sigmundova D., Iannotti R.J., Tynjälä J.A., Hamrik Z., Bucksch J. (2015) Secular trends in moderate-to-vigorous physical activity in 32 countries from 2002 to 2010: A cross-national perspective. Eur. J. Pub. Health, 25(suppl_2): 37-40.

29. Kibbe D.L., Hackett J., Hurley M., McFarland A., Schubert K.G., Schultz A., Harris S. (2011) Ten Years of TAKE 10 ! $®$ : Integrating physical activity with academic concepts in elementary school classrooms. Prev. Med., 52: S43-S50.

30. Kulinna P.H., Brusseau T., Cothran D., Tudor-Locke C. (2012) Changing school physical activity: An examination of individual school designed programs. J. Teach. Phys. Educ., 31: 113-130.

31. Russ L.B., Webster C.A., Beets M.W., Phillips D.S. (2015) Systematic review and meta-analysis of multicomponent interventions through schools to increase physical activity. J. Phys. Act. Health, 12: 1436-1446.

32. Vincent S.D., Sidman C.L. (2003) Determining measurement error in digital pedometers. Meas. Phys. Educ Exerc. Sci., 7: 19-24.

\section{Received 11.05.2018 \\ Accepted 27.08.2018}

(C) University of Physical Education, Warsaw, Poland 